\title{
Quantum Benchmark for Teleportation and Storage of Squeezed States
}

\author{
Gerardo Adesso $^{1,2}$ and Giulio Chiribella ${ }^{3}$ \\ ${ }^{I}$ Dipartimento di Matematica e Informatica, Universitá degli Studi di Salerno, Via Ponte Don Melillo, 84084 Fisciano (SA), Italy; \\ ${ }^{2}$ Grup de Física Teòrica, Universitat Autònoma de Barcelona, 08193 Bellaterra (Barcelona), Spain. \\ ${ }^{3}$ QUIT Quantum Information Theory Group and Dipartimento di Fisica "A. Volta", \\ University of Pavia, via A. Bassi 6, I-27100 Pavia, Italy
}

(Dated: April 2, 2008)

\begin{abstract}
We provide a quantum benchmark for teleportation and storage of single-mode squeezed states with zero displacement and completely unknown degree of squeezing along a given direction. For pure squeezed input states, a fidelity higher than $81.5 \%$ has to be attained in order to outperform any classical strategy based on estimation of the unknown squeezing and repreparation of squeezed states. For squeezed thermal input states, we derive an upper and a lower bound on the classical average fidelity, which tighten for moderate degree of mixedness. These results enable a critical discussion of recent experiments with squeezed light.
\end{abstract}

PACS numbers: 03.67.Hk

Quantum teleportation [1] and quantum state storage [2] are among the pillars of quantum information science, not only as groundbreaking demonstrations of the usefulness of quantum correlations against limited classical scenarios, but also as essential ingredients for quantum computation [3] and long-distance quantum communication [4]. Continuous variable (CV) systems [5], where quantum correlations arise between canonically conjugate observables (like the quadratures of light or the collective spin components of atomic ensembles), are especially suited for the unconditional implementation of these protocols [6]. While the seminal CV experiments only dealt with light fields [7], progresses towards the establishment of a complete quantum interface between light and matter have been recently undertaken, with milestones like the storage of coherent light onto atomic memories [8], and the teleportation from light to matter [9].

Quantum entanglement is the key resource that allows a sender (Alice) and a receiver (Bob) to beat any classical strategy for transmitting and storing quantum states. In realistic implementations, however, the quality of transfer/storage is limited by factors such as imperfections and losses. To decide whether an experiment demonstrates a genuinely quantum feature, one needs appropriate figures of merit and appropriate benchmarks in terms of them. The typical figure of merit characterizing quantum teleportation (from now on we restrict to this protocol, bearing in mind that our results equally apply to state storage too) is the fidelity [10] $\mathcal{F}=\left\{\operatorname{Tr}\left[\left(\sqrt{\varrho^{i n}} \varrho^{\text {out }} \sqrt{\varrho^{\text {in }}}\right)^{1 / 2}\right]\right\}^{2}$ between the unknown input state $\varrho^{\text {in }}$ to be teleported by Alice and the output state $\varrho^{\text {out }}$ which is actually obtained by Bob. To conclude that a quantum demonstration has taken place, the fidelity $\mathcal{F}$ has to beat the best possible fidelity $\mathcal{F}^{c l}$ —usually called classical fidelity threshold (CFT) — achievable by two cheating parties who have access to unlimited "classical" means (local operations and classical communication) but are not able to share entanglement, nor to directly transmit quantum systems [11]. Under this restriction, the only possibility for Alice and Bob is a "measure-and-prepare" strategy, where Alice measures the input system and communicates the outcome to Bob, who pre- pares the output according to her prescription. Devising the CFT is hence a problem of quantum estimation [12].

In the CV setting, the CFT has been only assessed for the special instance of pure coherent input states [11, 13]. In the limit case of completely unknown coherent amplitude, it yields the benchmark $\mathcal{F}_{c o h}^{c l}=1 / 2$, which has been extensively employed to validate experiments [7, 8, 9]. However, no such threshold is known for the case of squeezed states, which are currently drawing attention as input states of transfer protocols: the high-fidelity teleportation of squeezed states may enable cascading of teleportation, resulting in the construction of non-Gaussian gates (e.g. the cubic phase gate), useful to achieve universal CV quantum computation [6]. The lack of a CFT for squeezed states makes the validation of experiments a rather controversial issue, as there is no clear way to establish whether the achieved performances are signatures of a genuinely quantum information processing.

In this Letter we provide the first quantum benchmark for teleportation and storage of single-mode squeezed states with zero displacement and completely unknown degree of squeezing $r$ along a given direction. This encompasses the typical experimental situation [14] in which a squeezed state with known phase is generated, with the possibility of varying the degree of squeezing by tuning the nonlinearity of the optical parametric oscillator. For ideally pure input squeezed states (and pure squeezed reconstructed outputs [15]) the CFT reads $\mathcal{F}_{s q(\mu=1)}^{c l} \approx 81.5 \%$. We also address the search of a quantum benchmark for squeezed thermal states, which is necessary for a fair comparison with the actual experiments [16]. We show that a moderate amount of input mixedness only slightly modifies the classical average fidelity, rendering our benchmark robust against thermal noise. To prove this we provide an upper and a lower bound on the classical average fidelity, that coincide for pure input states and are close to each other for moderately mixed input states. Thanks to these results we provide a detailed discussion of recent experiments on teleportation and storage of squeezed (thermal) states [14, 17, 18], which appear on the edge to beat the upper bound, hence to pass the test of quantumness provided by our benchmark. 
We consider the transmission of single-mode non-displaced squeezed thermal states $\varrho_{r, \mu}^{i n}$ with purity $\mu \equiv \operatorname{Tr}\left[\varrho_{r, \mu}^{i n}{ }^{2}\right]$ and squeezing degree $r$ along a fixed direction (which we can assume to be the position axis without loss of generality [14]), defined by the action of the phase-free squeezing operator $\hat{U}(r)=\exp \left[\frac{r}{2}\left(\hat{a}^{\dagger 2}-\hat{a}^{2}\right)\right]$ on a thermal state $\varrho_{\mu}^{t h}=$ $(1-\Lambda) \sum_{n=0}^{\infty} \Lambda^{n}|n\rangle\langle n|$ [where $|n\rangle$ is the $n$ 'th Fock state, $\Lambda=(1-\mu) /(1+\mu)$ and the mean number of thermal photons is $\left.\bar{n}_{\mu}^{t h}=(1 / \mu-1) / 2\right]: \varrho_{r, \mu}^{i n}=\hat{U}(r) \varrho_{\mu}^{t h} \hat{U}(r)^{\dagger}$. For $\mu=1$ one recovers the pure squeezed vacuum state, $\left|\phi_{r}\right\rangle=\hat{U}(r)|0\rangle$. A squeezed thermal state, belonging to the family of Gaussian states [19], can be completely described by its covariance matrix $(\mathrm{CM}) \boldsymbol{\sigma}_{r, \mu}=(1 / \mu) \operatorname{diag}\{\exp (2 r), \exp (-2 r)\}$. We aim at computing the CFT for teleporting states of the form $\varrho_{r, \mu}^{i n}$, with given $\mu$, and completely unknown squeezing $r$. With the expression completely unknown squeezing we refer here to the scenario where a verifier Victor secretly chooses a value of the squeezing parameter, and asks Alice to transfer the corresponding state to Bob. The verifier will eventually assign to Alice and Bob a score equal to the fidelity between input and output state. The CFT is the maximum score that Alice and Bob can get in this game with a classical "measure-andprepare" strategy [15]. The verifier will always choose the less favourable state, forcing the two parties to adopt the strategy that maximizes the minimum score [12]. Such a strategy has to work equally well for any possible value of $r$ [20]. This scenario corresponds to the limit case in which the input states are drawn according to an ideally "flat" prior distribution of the unknown squeezing parameter, in analogy to the typical situation considered for coherent states [11, 13].

In order to devise the CFT for squeezed states we employ the techniques of Ref. [21], in which a method to explicitly devise the optimal quantum measurement to estimate a completely unknown squeezing transformation $\hat{U}(r)$ acting on an arbitrary pure single-mode state $|\psi\rangle$ was provided. Such an optimal measurement yields an estimate of the squeezing degree equal to $r+\delta$ with probability distribution $p_{\psi}^{o p t}(\delta)$ (independent on $r$ and peaked around $\delta=0$ ) given by

$$
\begin{aligned}
& p_{\psi}^{o p t}(\delta)=\left|\left\langle\psi \mid \eta_{\psi}(\delta)\right\rangle\right|^{2}, \\
& \left|\eta_{\psi}(\delta)\right\rangle=\frac{1}{\sqrt{2 \pi}} \int_{-\infty}^{+\infty} d \nu e^{-i \nu \delta} \frac{\Pi_{\nu}|\psi\rangle}{\sqrt{\left\langle\psi\left|\Pi_{\nu}\right| \psi\right\rangle}},
\end{aligned}
$$

where $\Pi_{\nu}=1 /(2 \pi) \int_{-\infty}^{+\infty} d \lambda e^{i \nu \lambda} \hat{U}(\lambda)$. Note that the optimal measurement depends on the state $|\psi\rangle$ : If the optimal measurement for the state $|\psi\rangle$ is used to estimate the squeezing on a different state $|\phi\rangle$ [22] one will generally have a suboptimal estimation, with probability distribution given by

$$
p_{\phi, \psi}(\delta)=\left|\left\langle\phi \mid \eta_{\psi}(\delta)\right\rangle\right|^{2}
$$

still depending only on $\delta$, but not necessarily peaked around $\delta=0$. The estimation in Eq. (1) is optimal for a whole class of different figures of merit [23]. In particular, the fidelity between two squeezed thermal states with CMs $\boldsymbol{\sigma}_{r, \mu}$ and $\boldsymbol{\sigma}_{r+\delta, \mu}$ belongs to this class for any given value of $\mu$. It depends only on the purity $\mu$ and on the difference $\delta$, and is given by [24] $\mathcal{F}_{\delta, \mu}=2 \mu^{2} /\left[\mu^{2}+\sqrt{\mu^{4}+2 \cosh (2 \delta) \mu^{2}+1}-1\right]$.

We discuss now the consequences of the above results for the transfer of phase-free squeezed states. The exact CFT for pure squeezed vacuum input states can be obtained by setting $|\psi\rangle=|0\rangle$ in Eq. (1), which yields the optimal probability distribution $p_{0}^{o p t}(\delta)=\left|\int_{-\infty}^{+\infty} \frac{e^{-i \nu \delta}}{(2 \pi)^{5 / 4}}\right| \Gamma(1 / 4+i \nu / 2)|d \nu|^{2}$, $\Gamma(z)$ being the Euler gamma function. The CFT is then given by the average fidelity of the optimal estimation strategy, and can be evaluated by numerical integration of the expression $\mathcal{F}_{s q(\mu=1)}^{c l}=\int d \delta p_{0}^{o p t}(\delta) \mathcal{F}_{\delta, \mu=1}$. This leads to the benchmark $\mathcal{F}_{s q(\mu=1)}^{c l} \approx 0.81517$, as anticipated. We notice that the CFT of $81.5 \%$ is sensibly higher than the corresponding one of $50 \%$ for coherent states [13]. This could be expected, as it is easier to estimate a single real parameter than a complex amplitude. This also shows that an experimental demonstration of genuine quantum features is much more demanding for squeezed states than for coherent states.

Unlike the case of coherent states (whose purity is unaffected by photon losses), to have a fair comparison with the experiments [14], it is crucial to investigate the CFT for input squeezed states which are realistically mixed [16]: this is a much harder estimation problem. In the following we derive both an upper and a lower bound on the average CFT for input squeezed thermal states $\varrho_{\mu, r}^{i n}$ with given purity $\mu$ (associated to the experimental losses) and completely unknown degree of squeezing $r$. With average CFT we mean the average over $\delta$ of the fidelity between $\varrho_{\mu, r}^{i n}$ and $\varrho_{\mu, r+\delta}$, the state prepared by Bob when estimating $r+\delta$ [15], maximized over all possible estimation strategies [25]. The two bounds individuate the value of the average CFT $\overline{\mathcal{F}}_{s q(\mu)}^{c l}$ within a window that gets as narrower as the purity of the state is higher, shrinking for $\mu \rightarrow 1$ onto the exact value of the CFT for pure states. Let us start with the upper bound, which is easily obtained by considering the ensemble decomposition of the thermal state as a mixture of Fock states. For any possible measurement performed on a squeezed thermal state the probability distribution will be $p_{t h}(\delta)=(1-\Lambda) \sum_{n=0}^{\infty} \Lambda^{n} p_{n}(\delta)$, where $p_{n}(\delta)$ is the probability distribution for the same measurement when performed on the squeezed Fock state $\hat{U}(r)|n\rangle$. If we consider the optimal estimation, with probability $\tilde{p}_{t h}(\delta)$, we obtain the bound: $\overline{\mathcal{F}}_{s q(\mu)}^{c l}=\int_{-\infty}^{+\infty} \tilde{p}_{t h}(\delta) \mathcal{F}_{\delta, \mu} d \delta=$ $(1-\Lambda) \sum_{n=0}^{\infty} \Lambda^{n}\left(\int_{-\infty}^{+\infty} \tilde{p}_{n}(\delta) \mathcal{F}_{\delta, \mu} \quad d \delta\right) \leq(1 \quad-$ $\Lambda) \sum_{n=0}^{\infty} \Lambda^{n}\left(\int_{-\infty}^{+\infty} p_{n}^{o p t}(\delta) \mathcal{F}_{\delta, \mu} d \delta\right) \equiv \overline{\mathcal{F}}_{s q(\mu)}^{u p}$, where $p_{n}^{o p t}(\delta)$ is the probability distribution of the estimation that is optimal for the Fock state $|n\rangle$, given by Eq. (1) with $|\psi\rangle=|n\rangle$. Explicitly, $p_{n}^{o p t}(\delta)=\left|\int_{-\infty}^{+\infty} \frac{e^{i \nu \delta}}{2 \pi} \sqrt{I_{n}^{\nu}} d \nu\right|^{2}$, where $I_{n}^{\nu}=\int_{-\infty}^{+\infty} d \lambda e^{i \lambda \nu}\langle n|\hat{U}(\lambda)| n\rangle$, and $\langle n|\hat{U}(\lambda)| n\rangle=$ $(\cosh \lambda)^{-n-1 / 2}{ }_{2} F_{1}\left[(1-n) / 2,-n / 2 ; 1 ;-\sinh ^{2} \lambda\right], \quad{ }_{2} F_{1}$ denoting the Gauss hypergeometric function. We stress that the upper bound is attained only for pure input states $(\Lambda \rightarrow 0)$ : for $\Lambda \neq 0$ one has $\overline{\mathcal{F}}_{s q(\mu)}^{c l}<\overline{\mathcal{F}}_{s q(\mu)}^{u p}$ strictly, as the optimal measurement of Eq. (1) depends on which state $|\psi\rangle$ 
is squeezed, and for a thermal ensemble there is no way to know which squeezed Fock state is measured.

On the other hand, any measurement performed by Alice automatically provides a lower bound on the CFT. We devise here a suitable estimation strategy working for squeezed thermal states. Observing that the squeezing transformation $\hat{U}(r)$ commutes with the parity operator $\hat{P}=\sum_{n}(-1)^{n}|n\rangle\langle n|$, to estimate $r$ we can perfectly separate the Fock states with even $n$ from those with odd $n$. The estimation strategy works as follows: (i) perform a Lüders measurement of the parity, thus projecting the squeezed thermal state onto the even/odd subspace: if the outcome is +1 , then the state will be proportional to $\sum_{n}^{e v e n} \Lambda^{n} \hat{U}(r)|n\rangle\left\langle n \| \hat{U}^{\dagger}(r)\right.$, otherwise it will be proportional to $\sum_{n}^{o d d} \Lambda^{n} \hat{U}(r)|n\rangle\langle n| \hat{U}^{\dagger}(r)$; (ii) for parity +1 (even subspace), perform the measurement that is optimal for the vacuum $|0\rangle$, otherwise perform the one that is optimal for the one-photon state $|1\rangle$. The probability distribution obtained with this strategy is $p_{t h}(\delta)=$ $(1-\Lambda)\left[\sum_{n}^{\text {even }} \Lambda^{n} p_{n, 0}(\delta)+\sum_{n}^{\text {odd }} \Lambda^{n} p_{n, 1}(\delta)\right]$, with $p_{n, 0}$ and $p_{n, 1}$ as in Eq. (2), and yields the bound: $\overline{\mathcal{F}}_{s q(\mu)}^{c l} \geq$ $\int_{-\infty}^{+\infty} p_{t h}(\delta) \mathcal{F}_{\delta, \mu} d \delta \equiv \overline{\mathcal{F}}_{s q(\mu)}^{l o}$. We notice that this bound also converges to the actual CFT for $\mu \rightarrow 1$.

The upper and lower bounds on the average CFT have been numerically evaluated for several values of input purity $\mu<1$, down to $\mu=1 / 9 \approx 0.11$, as plotted in Fig. 1] We notice that they are very close to each other if the input states are affected by a moderate amount of thermal noise, resulting in an error which is smaller than $2 \%$ in the experimentally relevant region of $\mu \geq 1 / 2$. This allows us to conclude that in this region the average CFT slightly decreases with decreasing $\mu$ compared to the benchmark at $\mu=1$. The two bounds become looser in the highly mixed regime, still allowing us to locate the average CFT between $70 \%$ and $90 \%$ for extremely thermalized input states ( $\mu$ in the vicinity of 0 ).

In our approach we have so far assumed the purity $\mu$ of the input thermal state to be perfectly known (requiring an exact knowledge of the experimental losses). However, by averaging our bounds over $\mu$ we can readily get bounds on the average CFT holding when the squeezed thermal states prepared by Alice and Bob have equal purity randomly distributed according to an arbitrary probability distribution $p(\mu)$. For example, for a flat distribution of input purity in the range $\epsilon \leq$ $\mu \leq 1, \epsilon=1 / 9$, we obtain the upper bound $\overline{\mathcal{F}}_{s q}^{c l}<81.3 \%$. This and similar bounds for $\epsilon \rightarrow 0$ can be used to discuss experiments in which both $r$ and $\mu$ are assumed to be completely unknown. The corresponding average CFT provides a test that has to be passed by truly quantum implementations, where $\mu$ and $r$ are not independent [16] (they seem related by an empirical law of the form $\mu \propto e^{-a|r|^{b}}$, with suitable parameters $a, b)$.

We now apply our results to the analysis of recent experiments involving teleportation and storage of squeezed states, as shown in Fig. 1. Two experiments dealt with teleportation of non-displaced squeezed thermal states of light with

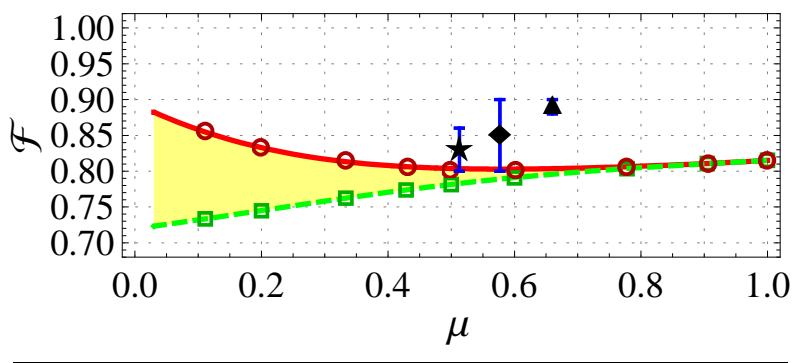

\begin{tabular}{cccccccccc}
\hline \hline$\mu$ & $1 / 9$ & $1 / 5$ & $1 / 3$ & $3 / 7$ & $1 / 2$ & $3 / 5$ & $7 / 9$ & $19 / 21$ & 1 \\
\hline$\overline{\mathcal{F}}_{s q(\mu)}^{u p}[\%]$ & 85.5 & 83.3 & 81.3 & 80.6 & 80.4 & 80.3 & 80.6 & 81.1 & 81.5 \\
$\overline{\mathcal{F}}_{s q(\mu)}^{l o}[\%]$ & 73.3 & 74.5 & 76.2 & 77.4 & 78.1 & 79.1 & 80.3 & 81.1 & 81.5 \\
\hline \hline
\end{tabular}

FIG. 1: (color online) Plot of the average CFT for the transfer of single-mode squeezed thermal states with purity $\mu$. Hollow circles (squares), whose coordinates are reported in the table, provide an upper (a lower) bound on the average CFT and admit a polynomial fit by the solid (dashed) line, with equation $\frac{4 \mu^{4}}{25}-\frac{11 \mu^{3}}{20}+\frac{3 \mu^{2}}{4}-\frac{11 \mu}{25}+$ $\frac{179}{200}$ (resp. $\frac{21 \mu^{4}}{200}-\frac{6 \mu^{3}}{25}+\frac{3 \mu^{2}}{25}+\frac{11 \mu}{100}+\frac{18}{25}$ ). Filled symbols correspond to the measured fidelities versus the purity of the produced states, achieved in recent demonstrations (as discussed later in the text): the diamond " $"[\mu \approx 0.58,|r| \approx 5.3 \mathrm{~dB}, \mathcal{F}=(85 \pm 5) \%]$, referring to [14], and the star “ $\star$ " [ $\mu \approx 0.51,|r| \approx 9.1 \mathrm{~dB}, \mathcal{F}=(83 \pm 3) \%]$, referring to [17], correspond to teleportation experiments; while the triangle " $\Delta$ " $[\mu \approx 0.66,|r| \approx 5.4 \mathrm{~dB}, \mathcal{F}=(89 \pm 1) \%]$ denotes the storage experiment of Ref. [18].

known phase [14, 17], achieving fidelities $\mathcal{F}=(85 \pm 5) \%$ at $\mu \approx 0.58$ [14], and $\mathcal{F}=(83 \pm 3) \%$ at $\mu \approx 0.51$ (here broadband squeezing was teleported) [17, 27], respectively. The expected values for the measured fidelities reasonably appear to pass our test, even though we judge that the challenge of a clear-cut demonstration of quantum teleportation of squeezed light, which likely appears within reach, is not closed yet. Regarding quantum memories, two recent experiments dealt with storage and retrieval of squeezed states using electromagnetically induced transparency [18, 28]. In particular, in [18] the fidelity $\mathcal{F}=(89 \pm 1) \%$ is reported for squeezed thermal input states with $\mu \approx 0.66$, neatly surpassing our upper bound on the average CFT.

We anyway stress that in all discussed experiments a single squeezed thermal state was teleported/stored. Unambiguous demonstration of a quantum transfer would require instead the CFT to be overcome by average experimental fidelities obtained in experiments involving several input states with a whole (ideally, infinite) range of values of $r$. In any experiment in which the input squeezing distribution is not ideally "flat", but has a realistically finite width, surpassing the value of our benchmark is a strictly necessary condition for demonstrating a quantum feature. This simple observation highlights the serious difficulties of the conventional CV teleportation protocol [26] (see also [5, 6, 7]), originally designed for coherent states, when employed for input squeezed states. Let us consider for simplicity the case of pure input states, with the entangled resource shared by Alice and Bob being a twin-beam Gaussian state with squeezing 
$s$, given by $\left|\Phi_{s}^{t b}\right\rangle_{A B}=\sum_{n=0}^{\infty}\left[\tanh ^{n}(s) / \cosh (s)\right]|n\rangle_{A}|n\rangle_{B}$. Then, the output of CV teleportation is still a Gaussian state and the fidelity between input and output is $\mathcal{F}^{Q}(r, s)=$ $\left\{2 e^{-2 s}[\cosh (2 r)+\cosh (2 s)]\right\}^{-1 / 2}$. We note that, for a given resource squeezing $s$ (a measure of the shared entanglement), $\mathcal{F}^{Q}(r, s)$ decreases with the input squeezing $|r|$, vanishing in the limit $|r| \rightarrow \infty$. Differently from the measure-andprepare strategy presented here, which works equally well for any value of $r$, the performances of the conventional quantum protocol drop exponentially with the amount of input squeezing. For any finite $s$ there is always a critical value $r_{c}$ beyond which the quantum protocol becomes less efficient than the classical strategy, $\mathcal{F}^{Q}\left(|r|>r_{c}, s\right)<\overline{\mathcal{F}}_{s q(\mu=1)}^{c l}$, despite the presence of entanglement. Moreover, using twin-beam resources with $s \leq 0.74(6.4 \mathrm{~dB})$, the fidelity of the teleported states is smaller than the CFT for any value of the input squeezing $r$. In short, the conventional $\mathrm{CV}$ teleportation scheme [26], while working excellently for coherent states (the fidelity does not depend on the complex amplitude, and the CFT of $50 \%$ is beaten iff entanglement is shared), is not really suited for the quantum transmission of squeezing.

The above discussion enables us to conclude that, no matter how efficient is the setup, no experiment using conventional teleportation can pass our test for all values $r$ of the squeezing. In view of this, the present benchmark serves as a "minimal test" that has to be passed in the finite range of values in which the experiment is designed to successfully work. The presented result strongly motivates the search of new schemes that are explicitly taylored for input squeezed states: it is very desirable to have covariant protocols that work equally well for any input squeezing, and among those to determine the optimal quantum strategy, able to beat the classical threshold as soon as entanglement is shared by Alice and Bob.

We warmly acknowledge discussions with A. Monras, A. Serafini, E. Polzik, L. Maccone, P. Perinotti, M. F. Sacchi, M. Aspachs, J. Calsamiglia, M. Piani, S. L. Braunstein, H. Yonezawa, A. Furusawa, and A. Lvovsky. GA was supported by Consolider-Ingenio 2010 CSD2006-0019 QOIT. GC was supported by the Italian Ministry MIUR through PRIN 2005.

[1] C. H. Bennett et al., Phys. Rev. Lett. 70, 1895 (1993).

[2] J. I. Cirac et al., Phys. Rev. Lett. 78, 3221 (1997).

[3] D. Gottesman and I. Chuang, Nature 402, 390 (1999).
[4] H. J. Briegel et al., Phys. Rev. Lett. 81, 5932 (1998).

[5] S. L. Braunstein and P. van Loock, Rev. Mod. Phys. 77, 513 (2005); N. Cerf, G. Leuchs, and E. S. Polzik (eds.), Quantum Information with Continuous Variables of Atoms and Light (Imperial College Press, London, 2007).

[6] A. Furusawa and N. Takei, Phys. Rep. 443, 97 (2007).

[7] A. Furusawa et al., Science 282, 706 (1998); W. P. Bowen et al., Phys. Rev. A 67, 032302 (2003); N. Takei et al., Phys. Rev. Lett. 94, 220502 (2005).

[8] B. Julsgaard et al. Nature 432, 482 (2004).

[9] J. F. Sherson et al., Nature 443, 557 (2006).

[10] A. Uhlmann, Rep. Math. Phys. 9, 273 (1976).

[11] S. L. Braunstein et al., J. Mod. Opt. 47, 267 (2000).

[12] A. S. Holevo, Probabilistic and Statistical Aspects of Quantum Theory (North Holland, Amsterdam, 1982).

[13] K. Hammerer et al., Phys. Rev. Lett. 94, 150503 (2005).

[14] N. Takei et al., Phys. Rev. A 72, 042304 (2005).

[15] In this Letter we always assume Alice's and Bob's labs to have the same ability in preparing quantum states: to reconstruct Alice's squeezed (thermal) states, Bob can only prepare squeezed (thermal) states. Allowing more freedom on Bob's side could further increase the CFT, leading to more demanding quantum benchmarks. A detailed discussion of such cases is beyond the scope of this work and will be provided elsewhere.

[16] For squeezed states, the higher the squeezing level is, the weaker the state becomes against losses. Such losses, associated to state preparation, can be experimentally estimated and modeled at first as input thermal noise.

[17] H. Yonezawa et al., Phys. Rev. Lett. 99, 110503 (2007).

[18] G. Appel et al., Phys. Rev. Lett. 100, 093602 (2008).

[19] G. Adesso and F. Illuminati, J. Phys. A 40, 7821 (2007).

[20] M. Ozawa, in Research Reports on Information Sciences, Series A: Mathematical Sciences, N. 74, Department of Information Sciences, Tokyo Institute of Technology (1980).

[21] G. Chiribella, G. M. D'Ariano, and M. F. Sacchi, Phys. Rev. A 73, 062103 (2006).

[22] The Dirac-normalized vectors $\left|\eta_{\psi}(\delta)\right\rangle$ provide a resolution of the identity in the subspace $\mathcal{H}_{\psi}$ spanned by the states $\hat{U}(r)|\psi\rangle$. The corresponding measurement can be performed on any state $|\phi\rangle \in \mathcal{H}_{\psi}$ (e.g. for $|\psi\rangle,|\phi\rangle$ Fock states with equal parity).

[23] This class contains all functions of $\delta$ with positive Fourier transform, in analogy to the Holevo class for phase estimation [12].

[24] H. Scutaru, J. Phys. A 31, 3659 (1998).

[25] Note that the average fidelity is smaller than the fidelity between $\varrho_{r, \mu}^{i n}$ and the average state prepared by Bob $\varrho_{\mu, r}^{a v e}=$ $\int p(\delta) \varrho_{\mu, r+\delta}$. Therefore the experimental fidelity in a quantum implementation must exceed our average CFT, as in Fig. 1.

[26] S.L. Braunstein and H.J. Kimble, Phys. Rev. Lett. 80, 869 (1998).

[27] H. Yonezawa, private communication.

[28] K. Honda et al., Phys. Rev. Lett. 100, 093601 (2008). 